\title{
The CST: its achievements and its connection to the light cone
}

Received: date / Accepted: date

\begin{abstract}
I review the applications of the Covariant Spectator Theory (CST) since its inception in 1969. Applications discussed here include calculations of $\mathrm{NN}$ scattering, $3 \mathrm{~N}$ bound states, electromagnetic form factors of few-nucleon systems, and the recent successes in describing the dynamical generation of quark mass and the meson spectrum using a chirially invariant quark-antiquark interaction that includes confinement. The common origin of the Light Cone technique and the CST, which dates back to the 1970's, will be discussed.
\end{abstract}

Keywords Covariant Spectator Theory $\cdot$ nuclear force $\cdot$ three body force $\cdot$ linear confinement $\cdot$ meson spectrum

\section{Background}

1.1 Context: two ways to approach non-perturbative calculations

If the goal is to solve QCD non-perturbatively, and to treat the one and two-body problems consistently, then we can identify two major approaches:

- Approach \#1 Start from the Dyson-Schwinger (DS) equation for the one-body self energy, and generalize to the two-body problem (c.f. Fig. 1), or

- Approach \#2 Start from the two-body Bethe-Salpeter (BS) equation, and construct the one-body equation (c.f. Fig. 2).

If we had a theory where the vertices or kernels could be calculated exactly to all orders, the two approaches would be equivalent, but if the vertices or kernels are modeled using phenomenological expressions, each of them has disadvantages. The disadvantage of approach \#1 (which starts from the one-body DS equation) is that its un-symmetric structure (only one of the vertices can be dressed if double counting is to be avoided) makes it less than straightforward how to extend it to the two-body sector without missing the dressing on the second vertex (new interaction terms are required). The

Many thanks to my collaborators who have worked with me over the years. Those who have contributed to the discussion covered in this mini-review include: Alfred Stadler, Teresa Peña, Elmar Biernat, J.W. Van Orden, D.O.Riska, Joe Milana, Cetin Savkli, M. Uzzo, Carl Carlson, John Tjon, Yohanes Surya, Gilberto Ramalho, and Sofia Leitão.

Franz Gross

Jefferson Laboratory

12000 Jefferson Avenue

Newport News, VA 23606, USA

Tel.: +1 (757) 869-1508

Fax: +1 (757) 269-7002

E-mail: gross@jlab.org 


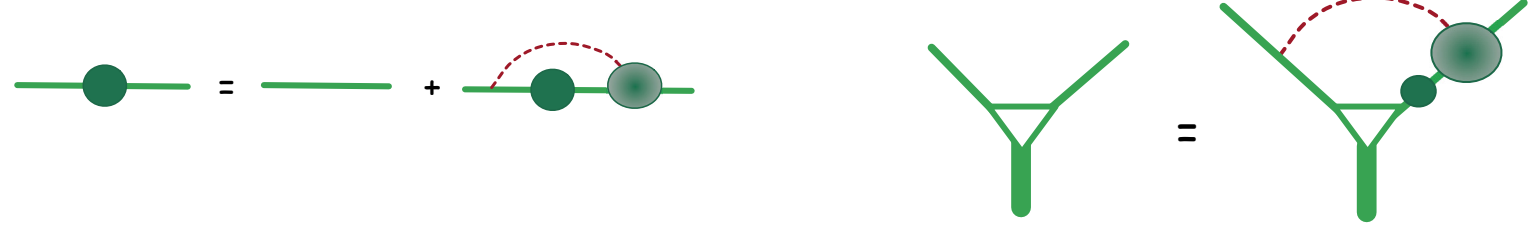

Fig. 1 (Left panel) one-body Dyson-Schwinger equation. (Right panel) attempt to generalize the one-body DS equation to describe two-body bound states
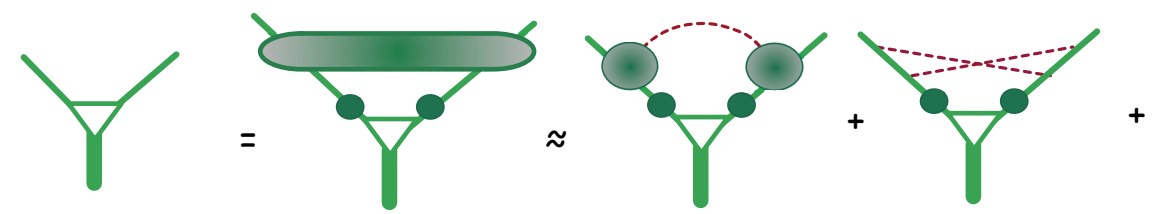

Fig. 2 Two-body Bethe-Salpter equation with ladder and crossed ladder approximations.

disadvantage of approach \#2 which starts from the two-body BS equation is that double counting may be introduced when the self-energy is calculated using the phenomenological two-body kernel. Spontaneous chiral symmetry breaking can be realized in both approaches, so this feature does not distinguish between them. The CST starts for the BS equation.

\subsection{Origin of the CST - from 1964}

Historical perspective In the early 1960's, field theory was not considered useful for non-perturbative calculations, and quarks and QCD had not yet been discovered. Strong interaction physics was the study of nucleon and meson (mostly pion) interactions, and dispersion theory (together with generalized unitary) was the only non-pertubative technique available. (With the emergence of QCD and the development of dynamical quark models, dispersion theory fell into disfavor for a period of time. It has recently been "rediscovered" as a useful tool.)

It was in this context that CST was developed. At that time I calculated the deuteron form factors using dispersion theory $[1 ; 2]$. Each of the three form factors could be written in the form

$$
F\left(Q^{2}\right)=\frac{1}{\pi} \int_{s_{0}}^{\infty} d s \frac{\operatorname{Im} F(s)}{s+Q^{2}}
$$

where $Q^{2}=-q^{2}$ with $q$ the four-momentum transferred by the virtual photon. The threshold $s_{0}$ is normally determined by the threshold for the physical production of the intermediate $N \bar{N}$ system (at $s_{0}=4 m^{2} \simeq 181.4 m_{\pi}^{2}$ ) that couples the photon to the deuteron-antideuteron final state (see the left panel of Fig. 3). This large threshold gives a very flat form factor that describes a very small deuteron,
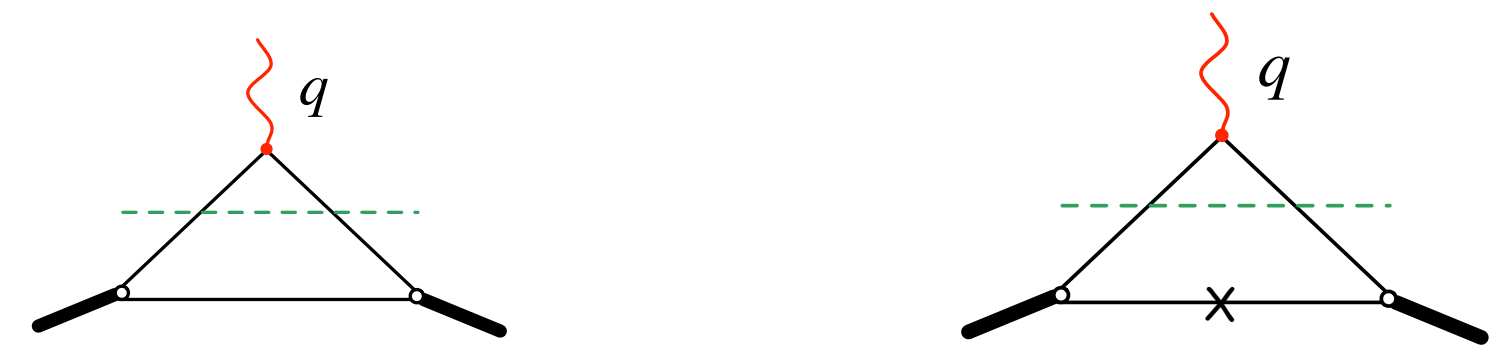

Fig. 3 ((Left panel) triangle diagram showing the normal two-body $N \bar{N}$ "cut" and (right panel) the anomalous cut where the "spectator" is on-shell. 

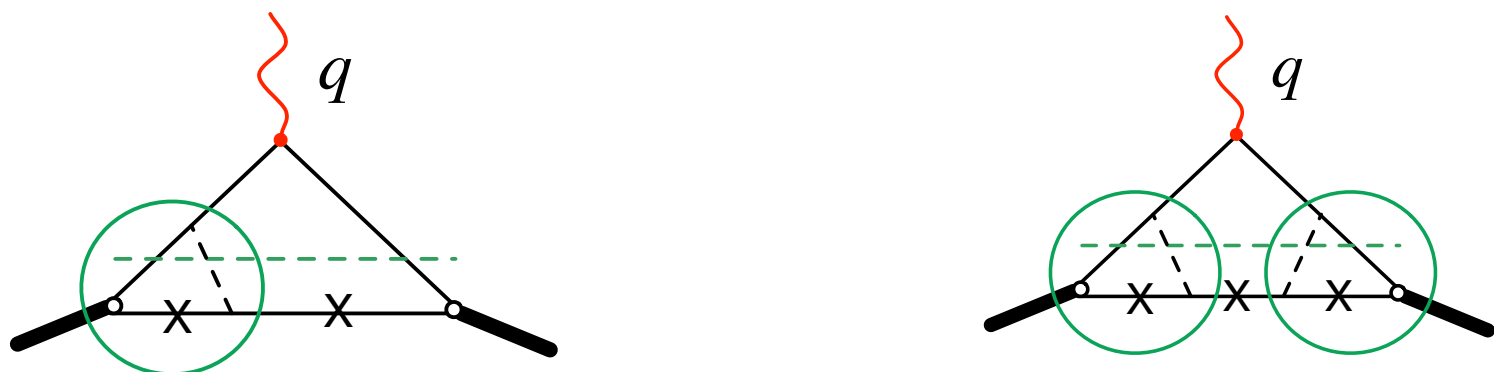

Fig. 4 (Left panel) anomalous region for the triangle diagram with a one-pion exchange (OPE) correction to the left vertex and (right panel) the anomalous region with OPE corrections at both vertices.

and is not in agreement with our picture of the deuteron as a weakly bound, extended system. To obtain the correct dispersion result for an extended deuteron with a mass $M_{d} \lesssim 2 m$, one treats $M_{d}$ as a complex variable and analytically continues the result for small $M_{d}$ to large $M_{d}$, being careful to stay on the same sheet. A careful study of this continuation [3] shows that when $M_{d}>\sqrt{2} m$, the dispersion integral must be deformed with a new threshold that migrates to a smaller value. If the deuteron binding energy $E_{B}$ is small, the new threshold is at $s_{0}=16 m E_{B} \simeq 1.73 m_{\pi}^{2}$, in complete agreement with our non-relativistic intuition for the behavior of a form factor of an extended, weakly bound system. Even more remarkable is the fact that the imaginary part of the form factor in the anomalous region (where $s_{0}<s<4 \mathrm{~m}^{2}$ ) is given by the imaginary part of the triangle diagram with the spectator on mass-shell, illustrated in the right panel of Fig. 3.

When pion exchanges at the vertices are included, the cut structure becomes more complicated, and the anomalous thresholds and anomalous regions show a pattern illustrated in Fig. 4. The thresholds for the one and two pion exchanges shown in Fig. 4 are given in Table 1. Note that these contributions

Table 1 Thresholds $s_{0}$ for the pion exchange diagrams shown in Fig. 4.

\begin{tabular}{lll}
\hline diagram & normal threshold & anomalous threshold \\
\hline one pion (left panel) & $(2 m+\pi)^{2} \simeq 209.3 m_{\pi}^{2}$ & $\simeq 11 m_{\pi}^{2}$ \\
two pions (right panel) & $(2 m+2 \pi)^{2} \simeq 239.2 m_{\pi}^{2}$ & $\simeq 28 m_{\pi}^{2}$ \\
\hline
\end{tabular}

can be summed by calculating a dressed $d p n$ vertex with one nucleon off-shell and the other nucleon restricted to its mass shell. When these contributions are summed to all orders, one has the spectator equation - this is the origin of the idea!

\subsection{Quick derivation of the CST for two-body scattering}

Start with the two-body Bethe-Salpeter equation for the scattering amplitude

$$
\mathcal{M}\left(p, p^{\prime} ; P\right)=\mathcal{V}\left(p, p^{\prime} ; P\right)-i \int \frac{d^{4} k}{(2 \pi)^{4}} \mathcal{V}(p, k ; P) S(k) S(P-k) \mathcal{M}\left(k, p^{\prime} ; P\right)
$$
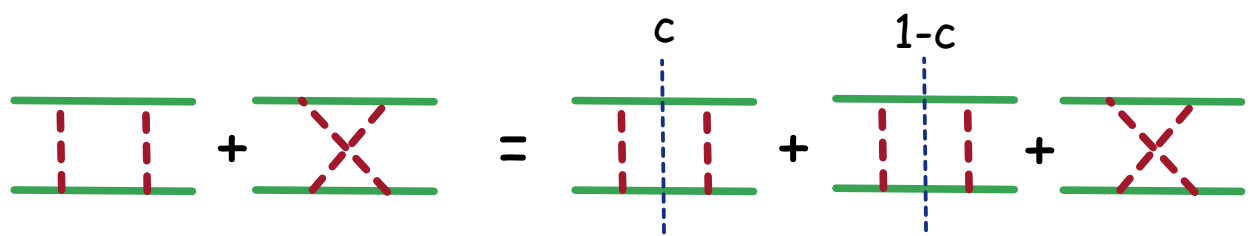

Fig. 5 The ladder and crossed ladders to 4th order. The ladder can be separated into two parts represented by the restriction "c" on the two-body propagator, and its remainder, " $1-c$ ". 
where $\mathcal{V}$ is the scattering kernel, $S$ the dressed one body propagator, $p, p^{\prime}, k$ are four-momenta of particle 1 , and $P$ the total four momentum. Unfortunately, the kernel is unknown and must be modeled. A common choice is to calculate the kernel from the infinite sum of all ladder and crossed ladder exchanges, illustrated to fourth order in Fig. 5. If the restriction "c" is chosen carefully, its remainder (" $1-c ")$ and the crossed box will be very small. If this cancellation works to all orders, then the two-body equation using the restricted two-body propagator with a one boson exchange (OBE) kernel will give a very good approximation to the BS equation using the full generalized ladder (ladder plus crossed ladder) exchanges.

It is commonly assumed that the choice $c=1$ (the BS in ladder approximation) is very good, but the crossed ladders are not small an this is not generally correct. In some cases, it can be proved that choosing $c$ to restrict one particle to its mass-shell (the heavier one if they have unequal masses) is more efficient [3;4], and in particular, this restriction is exact for scalar theories when the heavy (on shell particle) mass approaches infinity. This remarkable result provides a strong motivation for use of the CST.

\subsection{Comparison of the two-body CST with the two-body light-cone equation}

I close this background section by showing how the CST and the light cone equation are related. To see the connection, look at the 4th order box restricted to one of the two particles being on shell:

$$
\mathcal{V}_{4}=i \int \frac{d^{4} k}{(2 \pi)^{4}} \frac{\mathcal{V}(p, k ; P) \mathcal{V}\left(k, p^{\prime} ; P\right)}{D(k) D(P-k)} \rightarrow-\int \frac{d^{3} k}{(2 \pi)^{3}} \frac{\mathcal{V}(p, \hat{k} ; P) \mathcal{V}\left(\hat{k}, p^{\prime} ; P\right)}{2 E_{k} D(P-\hat{k})}=\mathcal{V}_{4}^{\mathrm{CST}}
$$

where $D(k)=m^{2}-k^{2}-i \epsilon, \hat{k}=\left\{E_{k}, \mathbf{k}\right\}, E_{k}=\sqrt{m^{2}+\mathbf{k}^{2}}$, and the decomposition is done in the rest frame $P=\{W, \mathbf{0}\}$, so that $D(P-\hat{k})=W\left(2 E_{k}-W\right)$.

The fourth order light cone result is computed in a similar way - by integrating over one of the components of $k$. Specifically, one decomposes $k$ into light cone variables using $k_{ \pm}=k_{0} \pm k_{z}$, so that

$$
\begin{aligned}
k & =\left\{k_{+}, k_{-}, \mathbf{k}_{\perp}\right\} \\
P & =\{W, W, \mathbf{0}\}
\end{aligned}
$$

and integrates over $k_{+}$keeping only the poles from the two particle propagators. Introducing the momentum fraction $x$ defined through $k_{-}=x P_{-}=x W$, gives

$$
\begin{aligned}
\mathcal{V}_{4} & =i W \int_{-\infty}^{\infty} d x \int \frac{d k_{+} d^{2} k_{\perp}}{2(2 \pi)^{4}} \frac{\mathcal{V}(p, k ; P) \mathcal{V}\left(k, p^{\prime} ; P\right)}{\left(E_{\perp}^{2}-W x k_{+}-i \epsilon\right)\left(E_{\perp}^{2}-W(1-x)\left(W-k_{+}\right)-i \epsilon\right)} \\
& \rightarrow-\int_{0}^{1} d x \int \frac{d^{2} k_{\perp}}{2(2 \pi)^{3}} \frac{\mathcal{V}\left(p, k_{\mathrm{LC}} ; P\right) \mathcal{V}\left(k_{\mathrm{LC}}, p^{\prime} ; P\right)}{m^{2}+\mathbf{k}_{\perp}^{2}-x(1-x) W^{2}}=\mathcal{V}_{4}^{\mathrm{LC}}
\end{aligned}
$$

where $k_{\mathrm{LC}}=\left\{\frac{m^{2}+\mathbf{k}_{\perp}^{2}}{x W}, x W, \mathbf{k}_{\perp}\right\}$. Note the famous result that the integral over $x$ is restricted to the interval $[0,1]$. This happens because outside of this interval the two poles from the propagator lie in the same half of the complex $k_{-}$plane, giving zero when the integral over $k_{-}$is evaluated using the Cauchy theorem.

At first the two results (3) and (5) appear to be unrelated, but introducing the variable $y$ and the transformations

$$
\begin{aligned}
& y=\frac{E_{k}-k_{z}}{W}=\frac{\hat{k}_{-}}{P_{-}} \quad \hat{k}=k_{\mathrm{LC}}^{\prime}=k_{\mathrm{LC}}(x \rightarrow y) \\
& W\left(2 E_{k}-W\right)=\frac{1}{y}\left[m^{2}+\mathbf{k}_{\perp}^{2}-y(1-y) W^{2}\right]
\end{aligned}
$$

were are able to transform $\mathcal{V}_{4}^{\mathrm{CST}}$ into

$$
\mathcal{V}_{4}^{\mathrm{CST}}=-\int_{0}^{\infty} d y \int \frac{d^{2} k_{\perp}}{2(2 \pi)^{3}} \frac{\mathcal{V}\left(p, k_{\mathrm{LC}}^{\prime} ; P\right) \mathcal{V}\left(k_{\mathrm{LC}}^{\prime}, p^{\prime} ; P\right)}{m^{2}+\mathbf{k}_{\perp}^{2}-y(1-y) W^{2}}
$$

Note that if we now change $y \rightarrow x,(7)$ is identical to (5) except that the range of the $x$ integration is now $[0, \infty)$ instead of $[0,1]$ (a profound difference, of course). This similarity showing the close connection between CST and LC has been noticed by several people over many years. 


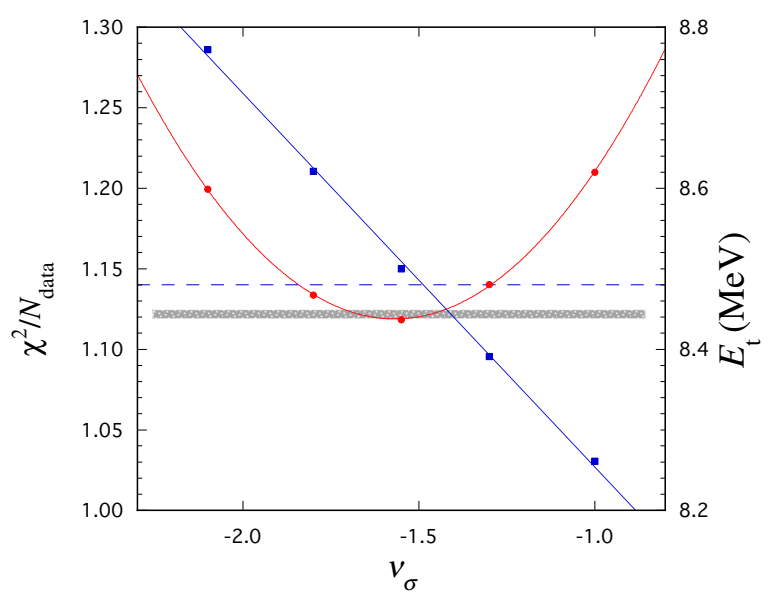

Fig. 6 Variation of $\chi^{2}$ from the fit to the two-body NN data (red line) and the triton binding energy $E_{t}$ (blue line), with the off-shell coupling $\nu_{\sigma}$ defined in Eq. (8). This figure is for model WJC-2 discovered in 2008, but is qualitatively similar to the result first discovered in 1997.

\section{Applications of the CST}

The CST has been used to study many systems:

- Few-Quark Systems (discussed in Sec. 2.2)

- Few-Nucleon Systems (discussed in Sec. 2.1)

- Atomic Systems (S. J. Brodsky and G. P. Lepage [5] and M. I. Edies, et.al. [6])

- Inelastic scattering (W. P. Ford, et.al. [7])

- Nucleon-Nucleus Scattering (K. M. Maung, et.al. [8])

- Nuclear Matter (L. S. Celenza and C. Shaken [9])

- Pion-Nucleon Scattering (Y. Sura and FG [10])

- Relativistic Explanation of the EMC Effect (S. Liuti and FG [11])

In this talk I will only have time to briefly review the first two topics.

\subsection{Applications of CST to Few-Nucleon Systems}

A few key papers in the use of CST for the study of few-nucleon systems are:

- 1969 (FG) First CST paper deriving the cancellation of ladder and crossed ladder exchanges for scalar and electromagnetic interactions [4]

- 1987 (FG and D. O. Riska) Derivation of a two-body current that is conserved, even if the interaction contains phenomenological form factors [12]

- 1995 (J. W. Van Orden, N. Devine, FG) Calculation of deuteron form factors using the first realistic CST wave functions [13

- 1997 (A. Stadler and FG) Observation that CST OBE models require no three-body forces. Demonstration that the fits to the NN data and the triton binding energy are very sensitive to the strength, $\nu_{\sigma}$, of the off-shell coupling of the $\sigma$ meson, and that the value of $\nu_{\sigma}$ that gives the best fit to the NN data also gives the correct triton binding energy, $E_{t}$ (see more detailed discussion below) [14]

- 1997 (A. N. Kvinikhidze and B. Blankleider) Three-body CST currents fixed by the gauging method $[15]$

- 2004 (FG, A. Stadler, and M. T. Peña) For CST OBE models, a conserved three-body interaction current is derived that uses the same one-body and two-body interaction currents needed for the two-body system [16]

- 2008 (FG and A. Stadler) The CST OBE model (WJC-2) is found that gives a precise fit to $n p$ scattering data $\left(\chi^{2} / N_{\text {data }} \sim 1.1\right)$ and requires only 15 parameters from the exchange of the photon and only 6 strongly interacting bosons $b\left[I\left(J^{P}\right)\right]: \sigma\left[0\left(0^{+}\right)\right], \eta\left[0\left(0^{-}\right)\right], \pi\left[1\left(0^{-}\right)\right], \delta\left[1\left(0^{+}\right)\right], \omega\left[0\left(1^{-}\right)\right]$, and $\rho\left[1\left(1^{-}\right)\right]$. These more accurate models show the same correlation between the quality of fit to the NN data and the three-body binding energy, first observed in 1997 [17] 
- 2014 (FG) derivation of a unique isoscalar two-body interaction current [18]

There is only space to focus on one of the most remarkable results of these CST OBE studies, illustrated in Fig. 6. The off-shell sigma coupling constant appears in the generalized $\sigma N N$ coupling

$$
\Lambda^{\sigma}\left(p, p^{\prime}\right)=g_{\sigma} \mathbf{1}-\nu_{\sigma}\left[\Theta(p)+\Theta\left(p^{\prime}\right)\right]
$$

where $p\left(p^{\prime}\right)$ is the four-momenta of the outgoing (incoming) nucleon and $\Theta(p)=(m-\not p) /(2 m)$ is the off-shell nucleon projection operator. Note that the interaction is independent of $\nu_{\sigma}$ if the nucleon is on-shell.

\subsection{Applications of CST to Few-Quark Systems}

The major results for the applications of CST to few-quark systems are:

- 1991- 4 (FG and J. Milana) Initial studies of the meson spectrum with a relativistic generalization of confinement (for $L=0$ states only) [19]

- 1999 (M. Uzzo and FG) Study of the stability of vector and scalar interactions using various equations [20]

- 2001 (C. Savkli and FG) Implications of the relativistic treatment of confinement [21]

- 2008 - (G. Ramalho, et.al.) Phenomenological models of hadronic form factors and static moments $[22]$

- 2014 - (E. Biernat, et.al.) Self energies and chiral symmetry breaking in a conjugation invariant CST model $[23 ; 24 ; 25 ; 26]$

- 2017 - (S. Leitão, et.al) Meson spectrum with $L \neq 0$ and spin (see the talk elsewhere in these proceedings) $[27]$

In this section I will highlight a few of these results. I start by emphasizing that here are two different ways to model confinement in QCD:

1. Require that the quark propagator have no poles on the real axis, or

2. Allow quark propagators to have poles on the real axis (i.e. quarks can be on shell), but construct an interaction that is zero when two or more quarks are on-shell (so that no physical scattering is possible)

The nonrelativistic and the relativistic CST approaches both assume the latter, while the well known Dyson Schwinger approach assumes the former. It may turn out that while the language used by the two approaches is quite different, they give equivalent results when properly carried out to all orders; future study may resolve these issues.

To understand the CST approach to confinement, first consider a screened definition of the linear confining potential in coordinate space

$$
\tilde{V}_{L}(r)=-\lim _{\epsilon \rightarrow 0} \frac{\sigma}{\epsilon}\left(e^{-\epsilon r}-1\right)=\sigma r .
$$

Transforming this to momentum space gives

$$
V_{L}(\mathbf{q})=\int d^{3} r e^{i \mathbf{q} \cdot \mathbf{r}}=-8 \pi \sigma \lim _{\epsilon \rightarrow 0}\left[\frac{1}{\left(\mathbf{q}^{2}+\epsilon^{2}\right)^{2}}-\delta(\mathbf{q}) \int d^{3} q^{\prime} \frac{1}{\left(\mathbf{q}^{\prime 2}+\epsilon^{2}\right)^{2}}\right]
$$

Note that this satisfies the important constraint

$$
0=\int d^{3} q V_{L}(\mathbf{q}) \rightarrow \tilde{V}_{L}(0)
$$

and leads immediately to the bound state momentum space Schrödiger equation for linear confinement

$$
\left[\frac{\mathrm{p}^{2}}{2 m_{R}}-E\right] \Psi(\mathbf{p})=-\sigma \int \frac{d^{3} k}{\pi^{2}} \frac{\Psi(\mathbf{k})-\Psi(\mathbf{p})}{(\mathbf{p}-\mathbf{k})^{4}} .
$$



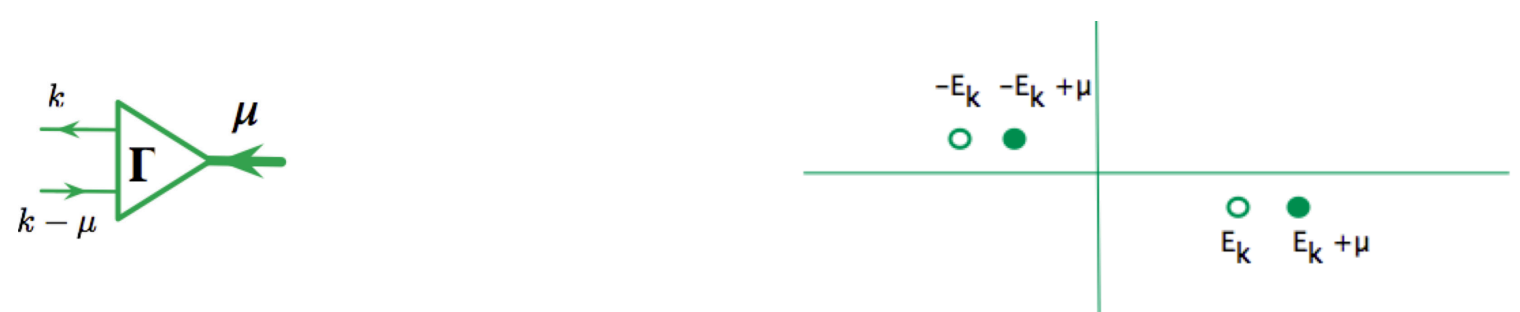

Fig. 7 (Left panel) the vertex function $\Gamma$ with bound state mass $\mu$ and the momenta of the particles labeled (here $\mu$ is a shorthand notation for the four-momentum $P=\{\mu, \mathbf{0}\}$ ). (Right panel) the location of the particle poles (16) in the complex $k_{0}$ plane.

This equation is regular and has been solved numerically, giving solutions identical to the well known coordinate space solutions. Modifications are required for $L \neq 0$ states [27].

These result are easily generalized for use with the one-channel CST equations. Labeling the momenta as in Eq. (2), it is convenient to generalize (10), using the on-shell particle 1 four-momentum in place of the nonrelativistic three-momentum, giving

$$
V_{L}(\hat{p}-\hat{k})=-8 \pi \sigma \lim _{\epsilon \rightarrow 0}\left\{\frac{1}{\left[(\hat{p}-\hat{k})^{2}-\epsilon^{2}\right]^{2}}-2 E_{k} \delta(\mathbf{p}-\mathbf{k}) \int \frac{d^{3} k^{\prime}}{2 E_{k^{\prime}}} \frac{1}{\left[\left(\hat{p}-\hat{k}^{\prime}\right)^{2}-\epsilon^{2}\right]^{2}}\right\} .
$$

Note that this reduces to (10) in the limit when the mass $m \rightarrow \infty$, and that it satisfies the relativistically invariant constraint

$$
0=\int d^{3} k \frac{m}{E_{k}} V_{L}(\hat{p}-\hat{k})
$$

which reduces to (11) in the nonrelativistic limit. The covariant one channel CST equation becomes

$$
\left[m^{2}-(P-\hat{p})^{2}\right] \Psi(\mathbf{p})=M_{b}\left(2 E_{p}-M_{b}\right)=-2 m \sigma \int \frac{d^{3} k}{\pi^{2}} \frac{m}{E_{k}} \frac{\Psi(\mathbf{k})-\Psi(\mathbf{p})}{(\hat{p}-\hat{k})^{4}} .
$$

These equations provide a convenient relativistic generalization of the linear confining potential, and their solutions have been shown to give a good qualitative description of the low lying $L=0$ states.

To study $q \bar{q}$ states which exhibit charge conjugation $(\mathcal{C})$ symmetric properties, it is necessary to generalize the one channel CST equation to two channels, and if the bound state mass (represented by $\mu$ in this discussion, so that in the rest system $P=\{\mu, \mathbf{0}\})$ is small, it must be extended to include all four possible channels. To understand how these coupled channel equations arise, examine the location of the four poles in the two-body propagator in the complex $k_{0}$ plane. In the rest system they are at:

$$
\begin{aligned}
S(k)^{-1} S(P-k)^{-1} & =\left(m^{2}-k^{2}-i \epsilon\right)\left(m^{2}-(P-k)^{2}-i \epsilon\right) \\
& =\left(E_{k}-k_{0}-i \epsilon\right)\left(E_{k}+k_{0}-i \epsilon\right)\left(E_{k}-\mu+k_{0}-i \epsilon\right)\left(E_{k}+\mu-k_{0}-i \epsilon\right)
\end{aligned}
$$

where the four-momenta of outgoing particle 1 is $k_{1}=k$, the four-momenta of the the incoming particle 2 is $k_{2}=k-P$ (or $-k_{2}=P-k$ when reinterpreted as an outgoing antiparticle), as illustrated in the right panel of Fig. 7, and for simplicity I have used propagators for scalar particles since the pole structure is independent of spin. The location of these poles in the complex $k_{0}$ plane is shown in the left panel of Fig. 7. If $\mu$ is large and the system is not $\mathcal{C}$ invariant, it is sufficient to keep only the pole at $k_{0}=E_{k}$ in the lower half plane, which restricts particle 1 to its positive energy mass shell. This is the one channel case I have discussed above.

When the system exhibits $\mathcal{C}$ invariance, it is necessary to construct CST equations that preserve this invariance. Since $\mathcal{C}$ transforms particles to antiparticles, the vertex function for a $q \bar{q}$ state is transformed from $\Gamma\left(k_{1}, k_{2}\right)=\Gamma(k, P)$ to $\Gamma\left(-k_{2},-k_{1}\right)=\Gamma(P-k, P)$ (where, for simplicity, I write the result only for scalar particles). Hence, when particle 1 is on-shell the vertex function is $\Gamma(\hat{k}, P)$, and under $\mathcal{C}$ it is transformed to $\Gamma(P-\hat{k}, P)$. This amplitude has $k_{2}=-\hat{k}$, and so corresponds to restricting the incoming particle 2 to is negative energy mass shell, and is not identical to $\Gamma(\hat{k}, P)$. The coupled equations shown in Fig. 8 couple these two different amplitudes together, ensuring that the 


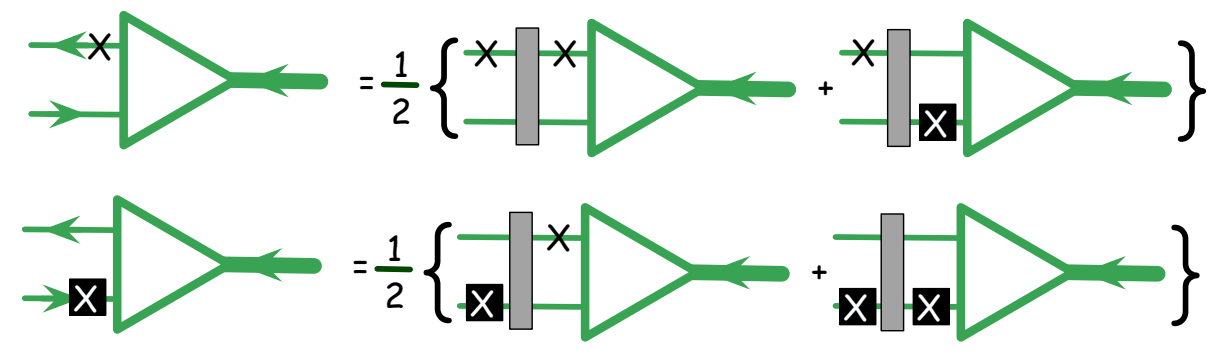

Fig. 8 The two-channel CST equation with exact charge conjugation symmetry. The particles marked with a $\times$ are on their positive energy mass shell and those with an $\times$ inside of a black square on their negative energy mass shell (see the discussion in the text). The triangles represent the vertex functions $\Gamma$.

system is $\mathcal{C}$ invariant. Operationally, the equations are obtained by averaging the contributions from the pole at $k_{0}=E_{k}$ in the lower half plane and the contribution from the pole at $k_{0}=-E_{k}+\mu$ in the upper half plane (hence a factor of $\frac{1}{2}$ is needed). Finally, if $\mu \rightarrow 0$, it is clear that none of the poles can be ignored, and in this limit a four channel equation is needed. For a detailed discussion, see Ref. [23].

Using the four channel equations, it has been shown what conditions must be met for spontaneous chiral symmetry breaking to occur [23]. If the kernel is a combination of scalar and vector interactions, so that it has the form $\mathcal{V}=V_{S} \mathbf{1} \otimes \mathbf{1}+V_{V} \gamma^{\mu} \otimes \gamma_{\mu}$, and the quark self energy is decomposed into $\Sigma(k)=A\left(k^{2}\right)+\not k B\left(k^{2}\right)$ (with $A_{0} \equiv A\left(m^{2}\right)$, etc.), spontaneous chiral symmetry breaking can only occur if two conditions are simultaneously satisfied

$$
\begin{aligned}
& 1=\frac{1+m_{0} / A_{0}}{4 m\left(1-B_{0}\right)^{2}} \int \frac{d^{3} k}{(2 \pi)^{2}} \frac{m}{E_{k}}\left[V_{V}(\hat{p}, \hat{k})+V_{V}(\hat{p},-\hat{k})+V_{S}(\hat{p}, \hat{k})+V_{S}(\hat{p},-\hat{k})\right] \\
& 1=\frac{1}{4 m\left(1-B_{0}\right)^{2}} \int \frac{d^{3} k}{(2 \pi)^{2}} \frac{m}{E_{k}}\left[V_{V}(\hat{p}, \hat{k})+V_{V}(\hat{p},-\hat{k})-V_{S}(\hat{p}, \hat{k})-V_{S}(\hat{p},-\hat{k})\right] .
\end{aligned}
$$

The first condition is a consequence of the requirement that the mass function $M\left(k^{2}\right)$ have a solution for a spontaneously generated mass $m$ (so that $M\left(m^{2}\right)=m$ ) and the second must be satisfied if the four-channel CST equations are to have a solution for a pion with zero mass. If the bare quark mass $m_{0}$ is zero, the equations are identical (signaling spontaneous chiral symmetry breaking) provided the constraint (14) is satisfied by the scalar part of the kernel. Hence, contrary to the normal intuition, a scalar interaction is consistent with the requirements of spontaneous chiral symmetry breaking provided it is purely confining.

\section{Summary and Outlook}

In conclusion, the CST

- is an efficient reorganization of the Feynman series,

- is manifestly covariant,

- has a smooth nonrelativistic limit which is easily interpreted

- provides a successful and efficient dynamical description of

- few-quark systems including spontaneous chiral symmetry breaking, quark self energies, and understanding of the meson spectrum, and

- few-nucleon systems including the ability to provide a precise fit to the $n p$ data base and the triton binding energy without the need for three-body forces .

Shortcomings of the CST (not discussed in this talk) include

- difficulties in extending it to new systems, where unexpected singularities can sometimes pose an obstacle,

- absence of a regularization scheme other than that based on the use of form factors, and

- the lack of a clear way to drive its equations from first principals. 
Acknowledgements This work was partially support by Jefferson Science Associates, LLC, under U.S. DOE Contract No. DE-AC05- 06OR23177.

\section{References}

1. Franz Gross (1964) Relativistic Calculation of the Deuteron Electromagnetic Form Factor I. Phys. Rev.134: B405

2. Franz Gross (1965) Relativistic Calculation of the Deuteron Electromagnetic Form Factor II. Phys. Rev.136: B140

3. F. Gross (1993) Relativistic Quantum Mechanics and Field Theory. Wiley Interscience, New York, 629 pages

4. F. Gross (1969) Three Dimensional Covariant Integral Equations for Low Energy Systems. Phys. Rev.186: 1448

5. S. J. Brodsky and G. P. Lepage (1977) Recent Advances in Bound State Quantum Electrodynamics. In: Fourth International Colloquium on Advanced Computing Methods in Theoretical Physics, Saint Maximin, France, March 21-23, 1977.

6. M. I. Edies, et.al. (2001) Theory Of Light Hydrogenlike Atoms. Physics Reports 342 (2001) 63-261, and private communication

7. W. P. Ford, S. Jeschonnek, and J. W. Van Orden (2014) Momentum distributions for ${ }^{2} \mathrm{H}\left(\mathrm{e}, \mathrm{e}^{\prime} \mathrm{p}\right)$. Phys.Rev. C90: 064006

8. K. M. Maung, et.al. (1991) Relativistic proton nucleus scattering and one boson exchange models. Phys.Rev. C43: $1378-1388$

9. L. S. Celenza and C. Shaken (1986) Relativistic Nuclear Physics: Theories of Structure and Scattering. World Scientific Publishers, Singapore

10. Y. Surya and F. Gross (1996) Unitary, Gauge Invariant, Relativistic Resonance Model for Pion Photoproduction. Phys. Rev. C 53: 2422

11. S. Liuti and F. Gross (1995) Extraction of the Ratio of the Neutron to Proton Structure Functions from Deep Inelastic Scattering. Phys. Letters 356B: 157

12. F. Gross and D. O. Riska (1987) Current Conservation and Interaction Currents in Relativistic Meson Theories. Phys. Rev. C 36, 1928

13. J. W. Van Orden, N. Devine, and F. Gross (1995) Elastic Electron Scattering from the Deuteron Using the Gross Equation. Phys. Rev. Letters 75: 4369

14. A. Stadler and F. Gross (1997) Relativistic calculation of the triton binding energy and its implications. Phys. Rev. Letters 78: 26

15. A. N. Kvinikhidze and B. Blankleider (1997) Gauging the three nucleon spectator equation. Phys.Rev. C56: 2973-2986

16. F. Gross, A. Stadler and M. T. Peña (2004) Relativistic calculation of the triton binding energy and its implications. Phys. Rev. C 69: 034007

17. F. Gross, and A. Stadler (2008) Covariant spectator theory of np scattering: Phase shifts obtained from precision fits to data below 350-MeV. Phys. Rev. C 78: 014005

18. F. Gross, Covariant Spectator Theory of np scattering: Isoscalar interaction currents. Phys. Rev. C 89: 064001

19. F. Gross and J. Milana (1994) Goldstone Pion and Other Mesons Using a Scalar Confining Interaction. Phys. Rev. D 50: 3332

20. M. Uzzo and F. Gross (1999) Stability of the Spectator, Dirac, and Salpeter equations for Mesons. Phys. Rev. C 59: 1009

21. C. Savkli and F. Gross (2001) Quark-antiquark bound states in the relativistic spectator formalism. Phys. Rev. C 63: 035208

22. F. Gross, G. Ramalho, and M.T. Peña (2012) Spin and angular momentum in the nucleon. Phys. Rev. D 85: 093006

23. E. Biernat, F. Gross, M. T. Peña, and A. Stadler (2014) Confinement, quark mass functions, and spontaneous chiral symmetry breaking in Minkowski space. Phys. Rev. D 89: 016005

24. E. Biernat, F. Gross, M. T. Peña, and A. Stadler (2014) Pion electromagnetic form factor in the Covariant Spectator Theory. Phys. Rev. D 89: 016006

25. E. Biernat, et.al. (2014) Chiral symmetry and $\pi \pi$ scattering in the Covariant Spectator Theory. Phys. Rev. D 90: 096008

26. E. Biernat, F. Gross, M. T. Peña, and A. Stadler (2015) Charge-conjugation symmetric complete impulse approximation for the pion electromagnetic form factor in the Covariant Spectator Theory. Phys. Rev. D 92: 076011

27. S. Leitão, A. Stadler, M. T. Peña, and E. Biernat (2017) Covariant Spectator Theory of heavy-light and heavy mesons and the predictive power of covariant interaction kernels. Phys. Lett. B764: 38-41 